\title{
The Method of Expert Evaluation of Airports Aviation Security Using Perceptual Calculations
}

\author{
Pavlenko Petro $^{1}$, Tavrov Dan ${ }^{2,3}$, Temnikov Volodymyr ${ }^{1}$, Zavgorodniy Sergiy $^{1}$, Temnikov Andrii ${ }^{1}$ \\ 1,3,4,5 National Aviation University, 1 Kosmonavta Komarova Ave., Kyiv, Ukraine, temnikov_v@ukr.net \\ ${ }^{2}$ Igor Sikorsky Kyiv Polytechnic Institute, 37 Peremohy Ave., Kyiv, Ukraine \\ ${ }^{3}$ Kyiv School of Economics, 92-94 Dmytrivska St., Kyiv, Ukraine, dtavrov@ kse.org.ua
}

\begin{abstract}
One of the effective ways to improve the quality of airport security (AS) is to improve the quality of management of the state of the system for countering acts of unlawful interference by intruders into the airports (SCAUI), which is a set of AS employees, technical systems and devices used for passenger screening, luggage, other operational procedures, as well as to protect the restricted areas of the airports. Proactive control of the SCAUI state includes ongoing conducting assessment of airport AS quality by experts, identification of SCAUI elements (functional state of AS employees, characteristics of technical systems and devices) that have a predominant influence on AS, and improvement of their performance. This article presents principles of the model and the method for conducting expert quality assessment of airport AS, whose application allows to increase the efficiency and quality of AS assessment by experts, and, consequently, the quality of SCAUI state control.
\end{abstract}

Keywords - aviation security, airport, acts of unlawful interference, system of counteraction to acts of unlawful interference, perceptual computer, type-2 fuzzy sets.

\section{INTRODUCTION (HEADING 1)}

Analysis shows that aviation security (AS) in many countries, including Ukraine, is at an unsatisfactory level [1-2], which is manifested, in particular, in the inability to prevent terrorist acts. According to [3-6], at present, the application of a set of technical measures, organizational measures, and measures aimed at reducing human factor are relevant for AS and aircraft safety.

One of the effective ways to improve the quality of AS is to improve the quality of the proactive control of the state of the system for countering acts of unlawful interference by intruders into airports (SCAUI), which is a combination of aviation security services (SS), technical systems and devices used in passenger screening, luggage, other operational procedures, as well as to protect restricted airport areas.

The sequence of actions for controlling the state of the SCAUI proposed by the authors is presented in Fig. 1.

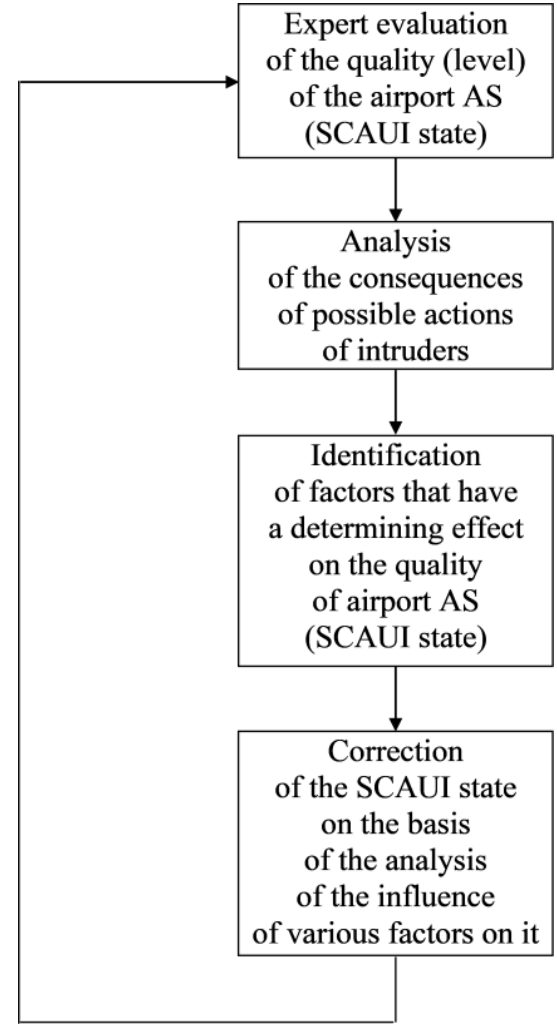

Fig.1. The sequence of actions for managing the condition of SCAUI

It should be noted that experts in the field of system reliability made an important contribution to the development of effective methods of expert evaluation. This is explained by the significant similarity of the problems and methods of solving them, which are dealt with by specialists working in the field of security and reliability [7-8].

Accounting for uncertainties is one such problem. Important results that allow to significantly reduce the influence of uncertainties in resolving the issues of increasing the reliability and safety of systems are given in [9-10]. 
To increase efficiency and quality of AS in the presence of uncertainty in expert assessments, the authors propose to use perceptual computing and the developed model of SCAUI, which together allow to fully take into account the features of the domain, such as the presentation of quality assessments by experts, the linguistic form of assessing the quality of individual operational procedures, technical equipment, and functional state of the SS officers, as well as the fact that the domain is very ill-defined.

\section{PERCEPTUAL COMPUTING AS A TOOL FOR EXPERT} ASSESSMENT OF THE QUALITY OF AVIATION SECURITY

\section{A. Motivation for applying perceptual computing}

The novelty of the developed methodology for conducting expert assessment of the quality of AS is the application of fuzzy set theory, in particular, computing with words and perceptual computing [11-12].

The quality of the airport AS depends on the functional state of the SS officers and the technical systems and devices they use when inspecting passengers and hand luggage, checking baggage, controlling passenger passes, etc. Due to the subjective nature of the concept of quality, AS should be modeled using linguistic concepts. In this article, we propose to model AS using perceptual computing.

Application of fuzzy sets allows to overcome uncertainties in the description of the domain related to subjective human thinking, and to obtain an assessment of the airport AS quality in a format understandable to a human decision maker. The use of perceptual computing is advisable given the fact that experts are able to assess the level of quality in a linguistic form, which greatly simplifies their work. Linguistic evaluation of the AS quality is natural, because, imprecisely defined concepts play an important role in human thinking [13].

As fuzzy sets, the authors propose to use interval fuzzy sets of type 2 (T2FS) [11], since they give an advantage when using linguistic variables and are the most common type of fuzzy sets of type 2 in practice.

The article proposes to evaluate the quality of performance of both various operational procedures and AS as a whole, enabling the experts to assess the quality of each SCAUI element (each aspect of AS) using words rather than precise numbers. Aggregation of these words, taking into account the relative importance of each SCAUI element (technical device or system) used in the implementation of the operational procedure and for the protection of the monitored airport zones, will give an assessment of the AS quality in a linguistic form.

The model of perceptual computing developed to evaluate the quality of AS is a model in a form of a loaded fuzzy oriented graph. Uncertainty is naturally caused by the uncertainty in assessments of the state of affairs in each node.

For the estimation of AS by linguistic indicators, the article uses an interval approach [11] that gives an opportunity to obtain an aggregated estimate of the AS quality according to local estimates given by many experts with different expertise in various aspects of AS.

\section{B. Fundamentals of Perceptual Computing}

Perceptual computing is a type of computing, in which objects of calculation are words and sentences expressed in natural language [14]. In other words, perceptual computing involves word-based calculations to create subjective assessments of the quality level [15].

When performing perceptual computing, words in a natural language are fed into the perceptual computer (Per-C), which allows a person to interact with the computer system using a vocabulary, i.e. a set of words modeled as T2FSs [16]. T2FSs are characterized by membership functions $\mu_{\tilde{A}}(x, u): X \times J_{x} \rightarrow[0,1]$, where $x$ is a primary variable, $u$ is a secondary variable with domain $J_{x} \subseteq[0,1]$ for each $x$, and $\mu_{\tilde{A}}(x, u)$ is a secondary grade of membership in $\tilde{A}$.

When applying perceptual computing, words are processed using the following three components [17]:

- encoder, which converts words to their T2FS representations that are then stored in a codebook;

- fuzzy set converter (CWW engine), which processes these T2FSs and forms one or more other T2FS outputs;

- decoder, which displays the output of the fuzzy set converter in a way understandable for a human.

\section{Encoder}

As mentioned above, each word in a codebook is modeled as interval T2FSs, for which secondary grades $\mu_{\tilde{A}}(x, u)=1 \quad \forall x \in X \quad \forall u \in J_{x}$, and the primary membership is the interval $J_{x}=\left[\underline{\mu}_{\tilde{A}}(x), \bar{\mu}_{\tilde{A}}(x)\right]$, where $\underline{\mu}_{\tilde{A}}(x)$ and $\bar{\mu}_{\tilde{A}}(x)$ are the lower (LMF) and upper (UMF) membership functions of $\tilde{A}$, respectively. The boundary between LMF and UMF, called the footprint of uncertainty $\operatorname{FOU}(\tilde{A})=\bigcup_{\forall x \in X} J_{x}$, represents uncertainty of $x$ 's membership in $\tilde{A}$. In the article, following recommendations in [17], words are represented using trapezoidal T2FS, for which LMF and UMF are trapezoidal membership functions:

$\mu_{\text {trap }}(x ; a, b, c, d, h)=\left\{\begin{array}{cc}(x-a) /(b-a), & a \leq x \leq b \\ h, & b \leq x \leq c \\ (d-x) /(d-c), & c \leq x \leq d \\ 0, & \text { otherwise }\end{array}\right.$

As such, each T2FS $\tilde{A}$ can be represented as a vector $(a, b, c, d, e, f, g, i, h)$ so that $\underline{\mu}_{\tilde{A}}(x)=\mu_{\text {trap }}(x ; e, f, g, i, h)$ 
and $\bar{\mu}_{\tilde{A}}(x)=\mu_{\text {trap }}(x ; a, b, c, d, 1)$. The graphic illustration (1) is shown in Fig. 2, where the shaded area is FOU.

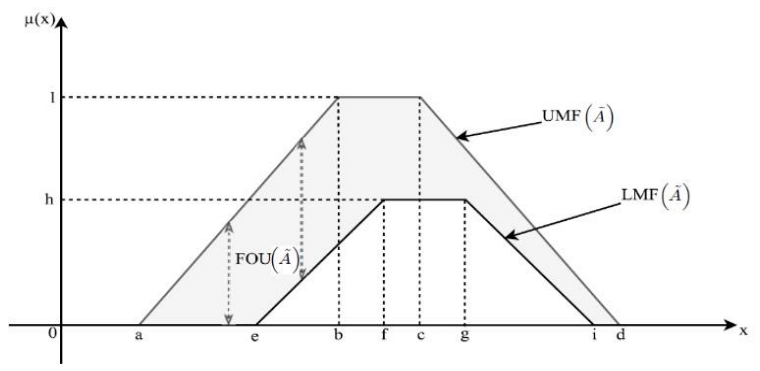

Fig.2. Trapezoidal interval fuzzy sets of type 2 [18]

\section{The $C W W$ Engine}

In this article, the authors use linguistically weighted mean values (LWA) [19-20], which, in the most general case, is expressed as

$$
Y_{L W A}=\frac{\sum_{i=1}^{n} X_{i} W_{i}}{\sum_{i=1}^{n} W_{i}}
$$

where each of the criteria for weighting and each weight are T2FSs. Algorithms for calculating LWA are omitted here due to space limitations and can be found in $[15,21]$.

\section{Decoder}

Decoder converts the output of the CWW engine into a Per-C output, which can be one of the following options [17]: a word from the codebook, most similar to the output of a fuzzy set converter, or a number of competing alternatives or a class.

\section{APPLICATION OF PERCEPTUAL COMPUTING TO DETERMINE THE QUALITY OF AVIATION SECURITY}

The decision-making process using the theory of perceptual computing for determining the quality of AS airports (SCAUI state) is shown in Fig. 3. This process is hierarchical and distributed, because it is performed on the basis of aggregation of independently made expert estimates of input factors AS.

The description of the decision-making process is based on the graphical hierarchical model of SCAUI, indicated in Fig. 3 by the dashed line. Note that in Fig. 3 a dashed line indicates the generalized version of the model of SCAUI, which can be presented in various forms, i.e. include various security factors depending on the actual circumstances.

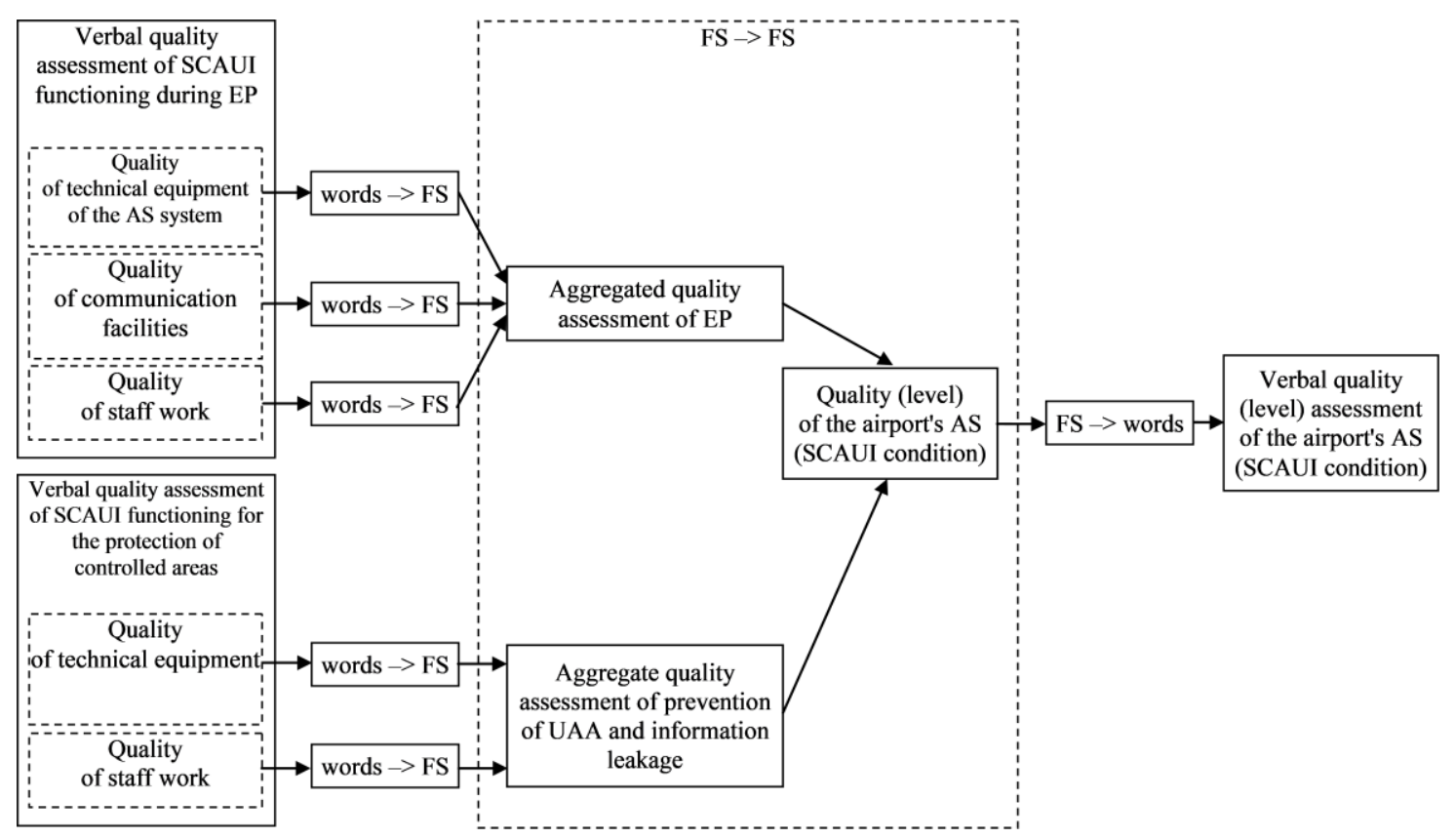

Fig.3. The decision-making process in applying perceptual computing to determine the quality of aviation security

The output level is the quality (level) of the airport AS, and the entry level consists of the parameters characterizing the functional state of SS officers, security systems of the airport and the technical devices used by for screening passengers, luggage, aircraft, and other actions to ensure the AS airport.
Elements of intermediate levels of the model are factors related to two groups:

- the quality of various operational procedures;

- the quality of preventing unauthorized access and leakage of information (organization and security of restricted areas of airports). 
These groups of factors correspond to basic and, at the same time, largely independent, airport security areas.

In Fig. 4, the process of arriving at an aggregated assessment of the AS quality is shown. It corresponds to the dashed "FS -> FS" box in Fig. 3.

Nodes of the graph are the elements of the process of organization and security of the airport. The model can have a relatively large (up to five or six) levels of the hierarchy. Nodes are connected with each other by directed loaded arcs characterizing the effect of the elements of one level of the model on the others. Weights are assigned by experts in a linguistic form using words from the Per-C codebook.

Input nodes A-I in Fig. 4 stand for factors that characterize the functional state of SS officers and the technical state of equipment used by SS (security systems and equipment used to inspect passengers and hand luggage, inspect baggage, monitor passenger passes, etc.).

Intermediate level consists of nodes $\mathrm{J}, \mathrm{K}$, and $\mathrm{L}$ that represent the quality of preflight checks of passengers and hand luggage, the quality of passport and ticket control, and the quality of preflight baggage inspection, respectively. Assessments of these quality levels are obtained as LWAs of appropriate lower-level node assessments using weights of the corresponding arcs.

The output node $\mathrm{W}$ is the quality of the preflight control of passengers and baggage. It is obtained as an LWA of assessments of nodes $\mathrm{J}-\mathrm{L}$, using the weights of the corresponding arcs. The output of the Per-C is, however, not the raw LWA, but the word from the codebook most similar to this LWA in terms of Jaccard's similarity metric [15].

\section{EXPERIMENTAL RESULTS}

To evaluate the proposed approach, the authors applied the method described above to a set of model data generated by the fourth author of the paper based on his vast experience in the domain.

The codebook consisted of five words representing linguistic assessments of each input node in the SCAUI model, and of five words representing weights of arcs in the model. Each word was defined on the interval $[0,1]$. For assessment, the following words were chosen: good $(\mathrm{G})$, normal $(\mathrm{N})$, satisfactory (S), acceptable (A), unsatisfactory (U). For weights, the following words were chosen: essential effect (Es), significant effect (Sg), average effect (Av), insignificant effect (In), little or no effect $(\mathrm{Lt})$

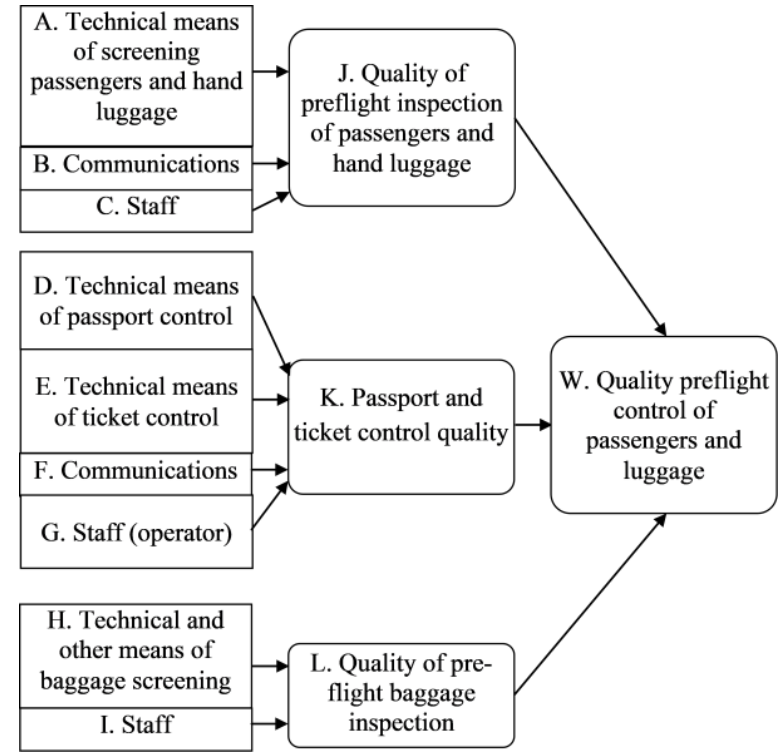

Fig.4. Fragment of the SCAUI model

Each word was converted to its IT2FS model using the interval approach [11]. Twenty experts in the field were surveyed and asked to provide intervals that, in their opinion, represent each word. The sets of intervals for each word were converted to a IT2FS model using an algorithm whose description lies outside the scope of this paper and can be found in [15]. In Table I, we present the obtained results (up to two significant digits).

TABLE I. Means $(m)$ and standard deviations $(\sigma)$ for left $(l)$ and right $(r)$ ends

of the intervals for each word from the codebook and their IT2FS models

\begin{tabular}{c|cccc|cccccccccc}
\hline \multirow{2}{*}{ Word } & \multicolumn{10}{|c|}{ Interval Statistics } & \multicolumn{1}{c}{ Parameters of Trapezoidal T2FS } \\
\cline { 2 - 14 } & $m_{l}$ & $\sigma_{l}$ & $m_{r}$ & $\sigma_{r}$ & $\boldsymbol{a}$ & $\boldsymbol{b}$ & $\boldsymbol{c}$ & $\boldsymbol{d}$ & $\boldsymbol{e}$ & $\boldsymbol{f}$ & $\boldsymbol{g}$ & $\boldsymbol{i}$ & $\boldsymbol{h}$ \\
\hline $\mathbf{G}$ & 0.88 & 0.04 & 1.00 & 0.00 & 0.74 & 0.98 & 1.00 & 1.00 & 0.93 & 1.00 & 1.00 & 1.00 & 1.00 \\
$\mathbf{N}$ & 0.74 & 0.03 & 0.87 & 0.04 & 0.66 & 0.77 & 0.83 & 0.93 & 0.74 & 0.80 & 0.80 & 0.86 & 0.68 \\
$\mathbf{S}$ & 0.63 & 0.03 & 0.73 & 0.03 & 0.56 & 0.65 & 0.71 & 0.83 & 0.65 & 0.68 & 0.68 & 0.71 & 0.45 \\
$\mathbf{A}$ & 0.54 & 0.04 & 0.62 & 0.03 & 0.48 & 0.55 & 0.61 & 0.68 & 0.55 & 0.58 & 0.58 & 0.61 & 0.49 \\
$\mathbf{U}$ & 0.00 & 0.00 & 0.53 & 0.04 & 0.00 & 0.00 & 0.05 & 0.78 & 0.00 & 0.00 & 0.04 & 0.65 & 1.00 \\
\hline $\mathbf{E s}$ & 0.88 & 0.04 & 1.00 & 0.00 & 0.74 & 0.98 & 1.00 & 1.00 & 0.93 & 1.00 & 1.00 & 1.00 & 1.00 \\
$\mathbf{S g}$ & 0.70 & 0.07 & 0.87 & 0.04 & 0.57 & 0.72 & 0.83 & 0.93 & 0.73 & 0.78 & 0.78 & 0.86 & 0.54 \\
$\mathbf{A v}$ & 0.46 & 0.10 & 0.69 & 0.07 & 0.24 & 0.48 & 0.68 & 0.84 & 0.52 & 0.58 & 0.58 & 0.64 & 0.37 \\
$\mathbf{I n}$ & 0.29 & 0.11 & 0.45 & 0.10 & 0.16 & 0.31 & 0.41 & 0.55 & 0.34 & 0.37 & 0.37 & 0.44 & 0.49 \\
$\mathbf{L t}$ & 0.00 & 0.00 & 0.28 & 0.11 & 0.00 & 0.00 & 0.04 & 0.53 & 0.00 & 0.00 & 0.01 & 0.13 & 1.00 \\
\hline
\end{tabular}


Weights for the arcs in the SCAUI model are given in Table II. They were assigned by the third author of this paper based on his expertise.

The Per-C with the described features was applied to a model dataset, in which, for each node, a linguistic assessment was provided as follows:

- A: good;

- B: good;

- C: normal;

- D: normal;

- E: normal;

- F: normal;

- G: normal;

- H: good;

- I: satisfactory.

TABLE II. Weights of arcs in the SCAUI model

\begin{tabular}{c|c|c}
\hline $\begin{array}{c}\text { Starting } \\
\text { Node }\end{array}$ & $\begin{array}{c}\text { End } \\
\text { Node }\end{array}$ & Weight \\
\hline $\mathrm{A}$ & $\mathrm{J}$ & $\mathrm{Es}$ \\
$\mathrm{B}$ & $\mathrm{J}$ & $\mathrm{Sg}$ \\
$\mathrm{C}$ & $\mathrm{J}$ & $\mathrm{Sg}$ \\
$\mathrm{D}$ & $\mathrm{K}$ & $\mathrm{Sg}$ \\
$\mathrm{E}$ & $\mathrm{K}$ & $\mathrm{Av}$ \\
$\mathrm{F}$ & $\mathrm{K}$ & $\mathrm{Av}$ \\
$\mathrm{G}$ & $\mathrm{K}$ & $\mathrm{Sg}$ \\
$\mathrm{H}$ & $\mathrm{L}$ & $\mathrm{Sg}$ \\
$\mathrm{I}$ & $\mathrm{L}$ & $\mathrm{Av}$ \\
$\mathrm{J}$ & $\mathrm{W}$ & $\mathrm{Es}$ \\
$\mathrm{K}$ & $\mathrm{W}$ & $\mathrm{In}$ \\
$\mathrm{L}$ & $\mathrm{W}$ & $\mathrm{Sg}$ \\
\hline
\end{tabular}

As a result, the following T2FS was obtained: (0.6633, 0.8510, 0.9116, 0.9731, 0.8183, 0.8520, 0.8980, $0.9185,0.3743)$. Using Jaccard's similarity metric, the following distances of this T2FS to each of the five words in the codebook were obtained:

- good: 0.3083;

- normal: 0.4388;

- satisfactory: 0.1296;

- acceptable: 0.0020;

- unsatisfactory: 0.0074 .

It can be concluded that the output of the system is the word "good," which captures the not so perfect state of the security for this given dataset.

\section{CONCLUSIONS}

The article presents the model developed by the authors and the method of implementing one of the most important stages of the proactive control of the SCAUI state, i.e. the expert assessment of the quality of aviation security of airports. The task of expert evaluation is the determination, with the help of experts, of the target value $\left[\hat{y}_{l}, \hat{y}_{r}\right]$ of the overall quality of the AS airport based on linguistic assessment by experts of the quality of each OP (each input factor $\mathrm{i}, i=1, \ldots, n$ ) required for aviation security.

\section{REFERENCES}

[1] Safety Report, International Civil Aviation Organization, Published in Montréal, Canada, 2017, 28 p.

[2] Safety review, State Aviation Administration of Ukraine, 2016, 25 p. (in Ukrainian).

[3] Doc 9859: Safety Management Manual, International Civil Aviation Organization, 3rd ed., 2013, 251 p.

[4] Doc 8973: Aviation Security Manual, International Civil Aviation Organization, 9th ed., 2014, 818 p.

[5] Doc 9808: Human Factors in Civil Aviation Security Operations, / International Civil Aviation Organization, 2002, 120 p.

[6] Annex 17: Security: Safeguarding International Civil Aviation Against Acts of Unlawful Interference, International Civil Aviation Organization, 2017, 62 p.

[7] E. Zio, "Reliability engineering: Old problems and new challenges", Reliability Engineering and System Safety, vol. 94, 2009, pp.125-141.

[8] T. Aven, and B. Heide, "Reliability and validity of risk analysis," Reliability Engineering and System Safety, vol. 94, no. 11, 2009, pp.1862-1868.

[9] E. Zaitseva, V. Levashenko, "Construction of a reliability structure function based on uncertain data," IEEE Tran on Reliability, 65(4), 2016, pp.1710 - 1723.

[10] T. Aven, E. Zio, P. Baraldi, and R. Flage, Uncertainty in Risk Assessment: The Representation and Treatment of Uncertainties by Probabilistic and Non-Probabilistic Methods, Wiley, 2014.

[11] F. Liu, J. M. Mendel, "Encoding words into interval type-2 fuzzy sets using an interval approach," IEEE transactions on fuzzy systems, no. 16, 2008, pp. 1503-1521.

[12] L. A. Zadeh, "Fuzzy logic = computing with words, " IEEE Trans. on Fuzzy Systems, vol. 4, 1996, pp. 103-111.

[13] L. A. Zadeh, "Fuzzy sets, " Information and control, vol. 8(3), 1965, pp. 338-353.

[14] L. A. Zadeh, "From computing with numbers to computing with words - from manipulation of measurements to manipulation of perceptions," IEEE transactions on circuits and systems I: Fundamental theory and applications 46(1), 1999, pp. 105-119.

[15] J. M. Mendel, "The perceptual computer: an architecture for computing with words," Proceedings of Modeling with Words Workshop in the Proceedings of FUZZ-IEEE 2001, pp. 35-38.

[16] J. M. Mendel, R. I. John, F. Liu, "Interval type-2 fuzzy logic systems made simple," IEEE transactions on fuzzy systems 14(6), 2006, pp. 808-821.

[17] J. M. Mendel, D. Wu, Perceptual computing. Aiding people in making subjective judgments. John Wiley \& Sons, Inc., Hoboken, New Jersey, 2010.

[18] D. Tavrov, L. Kovalchuk-Khymiuk, O. Temnikova, N.-M. Kaminskyi, "Perceptual Computer for Grading Mathematics Tests Within Bilingual Education Program," in Proceedings of The First International Conference on Computer Science, Engineering and Education Applications, 2018 (in press).

[19] D. Wu and J. M. Mendel, "Aggregation using the linguistic weighted average and interval type-2 fuzzy sets," IEEE Trans. on Fuzzy Systems, vol. 15, no. 6, 2007, pp. pp. 1145-1161.

[20] D. Wu and J. M. Mendel, "Corrections to "Aggregation using the linguistic weighted average and interval type-2 fuzzy sets'," IEEE Trans. on Fuzzy Systems, vol. 16, no. 6, 2008, pp. 1664-1666.

[21] D. Wu, J. M. Mendel, "Enhanced Karnik-Mendel algorithms," IEEE transactions on fuzzy systems 17(4), 2009, pp. 923-934. 